$\mathrm{T}$ he transmission of information and its translation into knowledge and new (or revised) policies and practices looms large in this first issue of Healthcare Quarterly for 2014, beginning with Ken Tremblay's discussion with Dr. Michael Schull, the CEO of the Institute for Clinical Evaluative Sciences (ICES). The evidence ICES generates is critical to policy-making by government, hospitals, health units and other "system stakeholders." Most exciting of all is Schull's commitment "to radically expand access to ICES data for publicly funded researchers" across Canada. What a brilliant makeover that will be.

\section{Managing Information}

Tremblay and Schull's conversation makes an apt preface for our first three articles, which centre on information management. We begin with Wayne Voisey Clark's needs-assessment study of electronic health records (EHRs) and the associated communication of personal health information among Manitoba Inuit. Applying a "two-eyed seeing" theoretical lens to his qualitative, participatory research, Clark developed a set of recommendations aimed at "increasing sustainability, authority and collaboration" in EHR management within the Inuit community.

Jeremy Theal and Denis Protti take us to a different corner of the information-management landscape: computerized physician order entry. Discussing the complex development and implementation of such a system at North York General Hospital (NYGH), Theal and Protti note that this project formed a major part of the organization's move towards evidence-based clinical practices. Candid about the resistance expressed, the authors document the manifold design details required to create a system that would both have physician buy-in and support NYGH's strategic priorities around quality and safety. In a future issue, we will publish part two of Theal and Protti's report documenting the project's success factors and outcomes.

In his interview mentioned above, Schull includes several comments on the need for much-improved "integration" across Canada's current healthcare scene. His views connect well to Karen Kuhn and Francis Lau's article on a web-based EHR system that facilitates inter-organizational information sharing. In their research, Kuhn and Lau found users perceived the view-only system as giving relevant and accurate information as well as enhancing information sharing and continuity of care. The major areas for dissatisfaction related to security and access issues - problems that must be addressed if EHR systems are to achieve their integrative potential.

\section{Quality Improvement}

From bringing together data, we shift to uniting service providers themselves. Justin Young and his co-authors address the origins, implementation and results of the Toronto Heart Attack Collaborative (THAC). This initiative combines community hospitals, percutaneous coronary intervention (PCI) centres and Toronto Emergency Medical Services (EMS) in an effort to guarantee patients across the city have access to PCI if they are suffering from ST elevation myocardial infarction. The new protocols, governance structures and funding approaches employed to organize and support THAC will interest readers in other jurisdictions, as will the undeniably positive results this multi-centre integration has wrought.

\section{Funding Reform}

Introduced in 2012, Ontario's Health System Funding Reform (HSFR) initiative moved the province's hospitals from "global" to 70-percent "patient-based" funding. At Toronto's Mount Sinai Hospital (MSH), Tyler Chalk and his co-authors report, that change was used as "a strategic basis for re-examining and redesigning operations" aimed at improving quality and efficiency. This "multidisciplinary collaborative effort" involved such things as ensuring clinical engagement and identifying best practices. Chalk et al. provide a useful case study (hip and knee replacements), and they put forth several lessons, including engaging key stakeholders and building consensus, overcoming "change fatigue," aligning with existing hospital initiatives focused on excellence and investing in support services (e.g., informatics).

\section{Applied Ethics}

Our next two articles appear under the rubric "applied ethics," because each one closely examines the direct impact of ethically informed decisions on specific, sometimes thorny, topics. In the first piece, Renaud Boulanger et al. examine the development of ethical decision-making among front-line staff in a network of home and community care agencies. Currently counting 42 organizational members, the Community Ethics Network (CEN) has generated in-house ethics policies, tool kits and guidelines on diverse topics, such as client choice, duty to care and staff safety. CEN's fostering of service providers' ethics capacity chimes with Jill Oliver and Paula Chidwick's report on three ethics quality improvement projects involving long-term care (LTC) facilities and aimed at minimizing errors that lead to unwanted and non-beneficial transfers of LTC residents back and forth to EDs. Oliver and Chidwick focus their discussion on decision-making and consent (e.g., by residents, by their substitutes). One striking finding is the immensely obstructive nature of level-of-care forms to ethical decision-making related to transfers.

\section{Case Management}

We conclude with an investigation of a side of healthcare decisionmaking that does not get as much press as it perhaps warrants: case management within an acute-care hospital environment. Kim Grootveld and three of her fellow case managers at Toronto's St. Michael's Hospital explore the contributions clinical-expert case managers - "the link between patient care and utilization management" - have made to improving patient care and resource use through "smart bed spacing" on St. Michael's General Internal Medicine (GIM) unit. Given the widespread interest in care coordination, financial management and process redesign these days in Canada and elsewhere, the sustainable, cost-effective steps and results Grootveld et al. address will be of interest to many Healthcare Quarterly readers. HQ 